\title{
Lateralization in the predatory behaviour of the common wall lizard (Podarcis muralis)
}

\author{
B. Bonati ${ }^{\mathrm{a}, *}$, D. Csermely ${ }^{\mathrm{a}}$, R. Romani ${ }^{\mathrm{b}}$ \\ a Dipartimento di Biologia Evolutiva e Funzionale, Sez. Museo di Storia Naturale, Università di Parma, via Farini, 90, 43100 Parma, Italy \\ b Dipartimento di Biologia Evolutiva e Funzionale, Sez. Biologia Animale, Università di Parma,viale Usberti 11A, 43100 Parma, Italy
}

\section{A R T I C L E I N F O}

\section{Article history:}

Received 28 March 2008

Received in revised form 10 July 2008

Accepted 13 July 2008

\section{Keywords:}

Behaviour

Common wall lizard

Podarcis muralis

Predation

Visual lateralization

\begin{abstract}
A B S T R A C T
Ectotherms have been shown being lateralized as well as mammals and birds. This is particularly evident in visual lateralization, i.e. the different use of the eyes, leading to use a specific eye to observe specific kind of stimuli and to process them with the correspondent contralateral hemisphere. Several lower vertebrates are facilitated in this from the lateral position of the eyes, enabling them to carry out more tasks simultaneously, controlled by different eyes and relative hemispheres. Predatory responses seem usually mediated by the right eye/left hemisphere in fishes, amphibians and some sauropsids, but there are no strong evidences of this in lizards. Eighteen wild males of the Common wall lizard Podarcis muralis were tested individually in captivity to ascertain whether they are lateralized to look at prey with a specific eye. The lizards were gently induced entering a 30-cm long central arm of a T-maze which led to a 44.5-cm long arm cross-arm at whose extremities there were two identical prey, Tenebrio molitor larvae, familiar to the lizards. We recorded what direction the lizards chose to reach the prey and the frequency and duration of head turning, indicative of looking either prey with the left or the right eye. We found that individuals show being lateralized at individual level. The preferred direction taken to reach the prey is the right for the majority of those ( 4 of 5) showing an evident preference, indicating also a possible form of laterality at population level. In addition, lizards maintained the same head side of the direction taken turned for more time towards the prey than the opposite head side, revealing an eye preference for observing this kind of cue. Our study demonstrates how males of Podarcis muralis have a visual lateralization to capture prey. Furthermore, it is another support to the hypothesis of vertebrate lateralization derivation from a common ancestor.
\end{abstract}

(c) 2008 Published by Elsevier B.V.

\section{Introduction}

Cerebral lateralization, i.e. the preferential use of one side of the body as a consequence of hemispherical specialization to control specific functions, has been studied in many mammals and birds, and in the past three decades in lower vertebrates too. The presence of lateralization in ectotherms, in fact, indicates that it is the expression of anatomical and consequent behavioural asymmetries. Thus, it is likely not the result of an evolutionary convergence, but a plesiomorphic character (e.g. Vallortigara and Bisazza, 2002). Many studies (e.g. Andrew, 1983; Rogers et al., 1985; Andrew and Dharmaretnam, 1993) have taken into consideration the likelihood that lateral-eyed animals, as are many ectotherms, are able to perform different tasks in response to a visual stimulus coming from either their left- or right-hand side. These animals also prefer to

\footnotetext{
* Corresponding author. Tel.: +390521 033 406; fax: +39 0521533673

E-mail address: beatrice.bonati@nemo.unipr.it (B. Bonati).
}

look at particular stimuli with either their left or their right eye, then process the stimuli with the contralateral hemisphere. As far as we know, this kind of visual asymmetry is effectively widespread among lateral-eyed ectotherms (Vallortigara et al., 1999a).

Independent eye use when observing the environment may lead to different brain elaboration of cues coming from different visual hemifields simultaneously. This allows the brain to avoid neuronal competition in the response to the stimuli, making the lateralization an efficient evolutionary solution for "functional incompatibility" (Sherry and Schachter, 1987).

Lizards are very interesting subjects in the study of lateralization, as the lateral position of their eyes minimizes binocular view and, similarly to other ectotherms, they do not have a large corpus callosum, which allows connection and communication between the two hemispheres. This is the case, for example, of Iguana iguana, where these connections are minimal (Butler and Northcutt, 1971). In Anolis lizards the visual system produces a hemisphere which is somewhat 'unaware' of what the other perceives, as a whole working as a "split brain" (Deckel, 1995). In anoles (Anolis carolinensis), 
and in male tree lizards (Urosaurus ornatus), aggression shows a left eye bias (Deckel, 1995; Hews and Worthington, 2001); in Sceloporus virgatus such phenomenon occurs in females too (Hews et al., 2004). Such a direction of lateralization for aggression tasks is widespread in different vertebrate taxa, in ectotherms, as in nonhuman primates (Vallortigara et al., 1999a; Hews and Worthington, 2001 ), suggesting that this behaviour is preferentially controlled by the right hemisphere.

A dissimilar situation is found in the predatory context, which seems to be right eye/left hemisphere mediated in several species of toads and bony fishes. In sauropsids our data are limited: we are aware of one study only, concerning a social group of the agamid lizard Ctnenophorus ornatus, where the right eye (left hemisphere) preference to control the predatory response seems to become stronger with familiarization to the prey (Robins et al., 2005). The habituation to the prey, therefore, could be important for the direction of visual lateralization, affecting the predatory cue codification. This reveals that there may exist an association between direction bias and experience, proving a complementary reptile brain specialization for processing different visual stimuli perceived from the close environment (Robins et al., 2005).

We aimed at ascertaining the possible existence of visual lateralization forms in the lacertid lizard Podarcis muralis in the predatory context. Our goal was to discover whether, when the lizard perceives two familiar prey with both eyes simultaneously in the monocular lateral field, it shows a preferential lateral direction, thus revealing a form of lateralization, and if this can be assessed at individual or population level, or both. We supposed that Podarcis muralis lizards, similarly to other ectotherms, are lateralized in the predatory response control, and we therefore expected a right eye/left hemisphere preference when observing the prey.

\section{Materials and methods}

From April to September 2007 we collected 18 adult male Podarcis muralis lizards from scattered small populations in Parma. They were captured by noose, a harmless and widespread capture method. They were then put in cloth bags and carried to the laboratory, where they were housed individually in $60 \mathrm{~cm} \times 50 \mathrm{~cm} \times 50 \mathrm{~cm}$ wood terraria. They had the front and one lateral side in glass, a $2 \mathrm{~mm} \times 2 \mathrm{~mm}$ wire mesh ceiling, floor covered with sand, one pebble and one brick for refuge and/or basking site; water was provided ad libitum. The terraria were located in a previous greenhouse, with full glass sides, but opaque roof; light, photoperiod and temperature were therefore natural, although artificial light and heat could be provided if necessary.

Once entering the terrarium, the lizards were fed with mealworm larvae (Tenebrio molitor), dusted with multivitamin powder. Here they remained for 1 week at least, being fed at 2-3 day intervals to accustom to the new environment and the food. This period was followed by 3 days of fasting before the tests (McKeehan and Sievert, 1996; Cooper, 2000; Shine, 2003) to induce and equalise the predatory motivation. At the end of each test-day the lizards were fed with one mealworm larva. The test sequence protocol lasted about 15 days and the lizards, therefore, remained in captivity for 3 weeks. At the end of the experimental period they were released at the same site of capture. None of them was harmed by the experiment, which was carried out under licence from Italian authorities.

The experimental apparatus consisted of one $8 \mathrm{~cm} \times 30 \mathrm{~cm} \times$ $6 \mathrm{~cm}$ and $8 \mathrm{~cm} \times 44.5 \mathrm{~cm} \times 6 \mathrm{~cm}$ PVC T-maze, covered with transparent and colourless plexiglas strips. The central arm of the maze had a rear entrance for the lizard, while the opposite end was the entrance to the cross-arm, which was prevented by a sliding sluice- gate, remotely operated by the observer by a cable. The cross-arm of the maze had one prey at both ends. The access to this arm of the maze was limited by two restriction blocks, which reduced the passage width to $4 \mathrm{~cm}$, to force the lizard having a straight head when entering the arm and then having the opportunity to see both prey with either eye at the same time, even though they might possess an even limited frontal binocular vision. This was to avoid that the lizard could choose either prey because seeing it first and then avoiding that the preference was not due to a form of lateralization. Light was homogeneous for the whole experimental apparatus and came from direct sunshine.

As prey we used two mealworm larvae (Tenebrio molitor), then already known to the lizards at testing. Not to influence the choice, we selected two identical prey by measuring their size putting them on graph paper and then freezing them, to avoid that their different movements could affect the lizard's choice. The position of the larvae was reversed after every test. A colourless, transparent plexiglas barrier before the prey prevented the lizard from ingesting the mealworm at the end of the test, in order to avoid that feeding could affect the motivation to prey in next test. Two new larvae were used at the beginning of each test-day.

At testing each individual was gently forced to enter the central arm of the maze, which was then closed. Here they remained for $15 \mathrm{~min}$. The test started when the sluice-gate was lifted up and ended when the lizard reached either prey, whereas it was considered void if the lizard refused to approach either prey within $20 \mathrm{~min}$. The test was recorded with a $2.5 \mathrm{~cm} \times 3.5 \mathrm{~cm}$ black and white video camera placed centrally in front of the maze and videotaped on a digital support. Videos were later reviewed using the "Virtualdub" video programme, which also permitted frame by frame analysis. The lizard behaviour was recorded continuously, using a digital event recorder. We considered the following behaviour parameters and relative occurrence:

- lateral direction chosen after entering the cross-arm of the maze.

- frequency and duration of head position in relation to the longitudinal body axis-right-side head exposure, left-side head turn, centred head position.

- frequency of tongue flicking.

- latency to the first movement.

- latency to enter the cross-arm.

- latency to the first attempt to prey.

Our aim was to repeat the tests 10 times maximum for each individual, with a minimum interval of 60 min between tests; however this was not achieved due to wide individual variation in responsiveness to testing. The maze floor was cleaned with ethylic alcohol before the beginning of every test to remove every chemical cue possibly influencing the subsequent choice. Each lizard was tested three times daily maximum and tested again after 3 days. The number of daily tests could be variable due to meteorological or temperature conditions. Maze floor temperature was measured with a $5.0 \mathrm{~cm} \times 2.2 \mathrm{~cm} \times 1 \mathrm{~cm}$ temperature data-logger fixed to the experimental apparatus, with data downloaded with MTT Tempstick software.

We used the Kruskal-Wallis ANOVA $(K W)$, to compare durations and tongue-flicking frequency among the individuals, and the Mann-Whitney $U$-test $(U)$, to compare the head orientation duration and the final preference, both calculated with the SPSS 14.0 for Windows software (SPSS Inc., 2006). The binomial test was used to ascertain the lateralization at the individual level, whereas the $\chi^{2}$-component " $z$ " index $(z)$ (Bishop et al., 1975 ) to compare the sample's total number of choice for either cross-arm and the tongue-flicking frequency for each side head exposition. Means are listed \pm S.E. throughout and the probabil- 
ity, set at $\alpha=0.05$, is two-tailed throughout, unless otherwise stated.

\section{Results}

We tested 18 male lizards which carried out 4-10 tests each (Table 1). The floor temperature during the tests was $31.0 \pm 2.4{ }^{\circ} \mathrm{C}$. After lifting the sluice-gate the lizard remained motionless and moved for the first time after $42.7 \pm 81.2 \mathrm{~s}$, but with great variability among the individuals $(K W=35.016, n=18, P<0.01)$. They moved within the central arm of the maze for a mean duration of $108.0 \pm 124.5 \mathrm{~s}$ before entering the cross-arm of the maze. In this case too the lizards showed wide variation $(K W=42.234, n=18$, $P<0.01$ ).

When arrived to the cross-arm they turned the head for the first time 59 times to the left-hand side and 75 times to the righthand side $(z=0.977, P>0.05)$. Considering the head orientations as a whole, the lizards kept the head along the body axis 185 times (grand total), whereas turned the head to the left-hand side 177 times total and to the right-hand side 158 times total. Considering the first test only the head orientation was turned 23 total times aligned to the body axis, 16 times to the left-hand side and 15 times to the right-hand side. At that location, the lizards remained for $2.3 \pm 2.5 \mathrm{~s}$ keeping the head aligned to the body axis, $2.5 \pm 3.3 \mathrm{~s}$ turning the head to the left-hand side and $2.44 \pm 1.8$ to the righthand side, but with high individual variability $(K W=9.659, n=3$, $P<0.01$ ). Such a duration was rather different among the individuals also for both the head kept aligned to the body axis and turned to the left-hand side $(K W=30.620, n=18, P<0.05$ and $K W=36.172$, $n=18, P<0.01$, respectively), but not for when turned to the righthand side $(K W=19.926, n=18, P>0.05)$. By comparison, during the first test these durations were homogeneous $(K W=19.731, n=18$, $P>0.05, K W=22.780, n=18, P>0.05, K W=20.075, n=18, P>0.05$, respectively).

When the lizards decided to approach to catch the prey found on their right-hand side, they mostly spent longer time keeping the head turned to the right more than keeping the head turned to the left $(U=5.670, n=72, P<0.001)$. In contrast, when the lizards attempted to catch the prey at their left-hand side, we recorded exactly the opposite phenomenon, i.e. the lizards kept the head turned to their left-hand side more than to the right-hand side $(U=5.509, n=79, P<0.001)$. Considering the first test only, when

Table 1

The results of binomial test for every individual, concerning the number of tests when lizard approached the prey at the left or right side of the cross-arm of the T-maze

\begin{tabular}{rccc}
\hline Lizard & Left & Right & $P$ \\
\hline 1 & 5 & 3 & $>0.10$ \\
2 & 1 & 9 & $<0.01$ \\
3 & 1 & 9 & $<0.01$ \\
4 & 5 & 5 & $>0.10$ \\
5 & 7 & 2 & $=0.07$ \\
6 & 2 & 8 & $<0.05$ \\
7 & 3 & 3 & $>0.10$ \\
8 & 1 & 3 & $>0.10$ \\
9 & 1 & 7 & $<0.05$ \\
10 & 7 & 3 & $>0.10$ \\
11 & 9 & 1 & $<0.01$ \\
12 & 5 & 3 & $>0.10$ \\
13 & 3 & 7 & $>0.10$ \\
14 & 5 & 4 & $>0.10$ \\
15 & 4 & 1 & $>0.10$ \\
16 & 2 & 3 & $>0.10$ \\
17 & 2 & 3 & $>0.10$ \\
18 & 2 & 2 & $>0.10$ \\
Total & 65 & 76 & \\
\hline
\end{tabular}

the lizards moved to the prey on their left-hand side they kept their head turned to the left longer than to the right $(U=2.386$, $n=5, P<0.05$ ) while when they moved to the prey on the righthand side they kept the head turned to the right longer then to the left $(U=2.122, n=13, P<0.05)$.

Only 12 lizards performed at least 8 tests. Restricting our attention to these individuals, we found that 5 individuals only (42\%) were clearly lateralized, as they were those showing a significant preference (binomial test, $P<0.05$ ) for the same direction in most of their tests.

Analysing the population as a whole, the lizards chose the right side of the cross-arm 76 times total vs. 65 total when they chose the left side $(z=0.655, P>0.05)$. However, considering the result of the first test in all the lizards, we get a preference for the right side (13R: $5 \mathrm{~L}$; binomial test, $P<0.05$ ). Moreover, if we consider the direction chosen by the lateralized lizards only, we find that 4 of 5 (80\%) strongly preferred the right direction (binomial test, $P<0.01$, $P<0.01, P<0.05, P<0.05)$, whereas the remaining one preferred the left direction (binomial test, $P<0.01$ ).

The lizards attempted to prey after a latency of $120.9 \pm 124.2 \mathrm{~s}$ (range 6-757s), but, again, with high individual variability $(K W=34.326, n=18, P<0.01)$. Tongue-flicking was performed $5.18 \pm 4.78$ times per test, again with high variability among the individuals $(K W=58.570, n=18, P<0,001)$. In particular, they tongue-flicked $2.1 \pm 0.3$ times with a right side and $1.6 \pm 0.2$ with a left side head exposition (298R: 226L total times; $z=2.224 ; P<0.01$ ).

\section{Discussion}

Our results show that lizards are individually lateralized, preferring a specific side to look at familiar prey and making for it, although the percentage of lateralization at the population level is quite low. Nevertheless, the lateralized individuals have a strong preference for a specific head direction indicative of eye exposure towards the prey. We suggest that the low percentage of lateralized lizards in the population is due to the small number of tests we were able to carry out for some lizards more than to a real absence of lateralization itself. Evaluating our data, in fact, we can note that the increase in number of individual trials leads to increase the tendency to choose a specific direction (Table 1). If we consider only individuals which performed at least 8 tests we obtain a greater proportion of lateralized individuals, 5 out of 12 .

The direction of visual lateralization for the predation task is the right. The low number of individuals really lateralized does not permit to assess this as a strong preference. In accord to this direction, however, the first choice of lizards in each test, that was constantly for the right direction.

Furthermore, the duration of right-side head exposure is the only parameter considered which was performed similarly by the lizards; in fact, they exposed the left side of the head as well as maintained the head aligned to the body axis with high variation. This can be indicative that lizards behaved differently when choosing to approach the prey using the left visual hemifield or the binocular one, but they behaved without individual difference when using the right visual hemifield. In fact, the durations of the right eye use towards the prey are similar among the individuals, whereas very variable for the left eye use.

In addition, there was a strong relation between the duration of eye exposure to the prey and the subsequent direction chosen to approach the prey: lizards invariably moved from the cross-arm towards the prey to which side they viewed for longer, showing that they prefer observing the prey with a specific eye. Furthermore, this is a confirmation of how a visual asymmetry may affect 
motor behaviour, contributing to ascertain the perceptual or motor origin of the lateralization (Vallortigara et al., 1999a). That result is similar to the detour tests conducted on chicks by Vallortigara et al. (1999b) and on several species of fishes by Bisazza et al. (1997a,b).

As a whole, our results reveal that Wall lizard males are lateralized because they observe the prey with a preferential eye (and then process the relative information with the contralateral hemisphere); in addition, the direction of such a lateralization (the right) is consistent within the population. This is in accord with previous studies, which revealed that in the vertebrate brain the left hemisphere (i.e. the right eye) controls detailed feeding responses, being correlated with the relative decisions, and that the predatory behaviour itself is then lateralized (Vallortigara et al., 1998; Robins and Rogers, 2004; Vallortigara and Rogers, 2005; Robins and Rogers, 2006).

However, literature information of that phenomenon is rather poor for sauropsids, apart from a study by Robins et al. (2005) on a social group of Ctenophorus ornatus. According to our results, those lizards are specialized to observe the prey within the right visual hemifield and processing that perceptual cue with the left hemisphere. In addition, in Ctenophorus ornatus the preference increased along the familiarization with prey, as also found in other vertebrates, such as toads (Robins, 2005; Robins and Rogers, 2006), where the brain control of visual cues from novel or familiar prey seems processed by different hemispheres, then leading to different directions of the lateralization output. This result can be related to experience and long-term memory, which may cause a switch of hemifield conducting the same kind of cues (Robins and Rogers, 2006). Wild-caught Brachyraphis episcopi females shown that there is a different response in individuals from high and low predation areas when facing a detour test (Brown et al., 2007). Brown et al. (2007), in fact, found that the $45 \%$ of the population of fishes exposed to high predatory pressure are strongly lateralized compared to just $15 \%$ of those exposed to low predatory pressure.

The form of lateralization ascertained in this study, therefore, likely evolved as an adaptive character in response to environments needing the performance of more tasks at the same time. It was necessary for Podarcis muralis lizards to monitor the surroundings while performing daily vital activities, such as feeding, in high predation pressure contexts. Knowing that experience could determine a different brain processing of stimuli, we offered the lizards only known prey. The low lateralization percentage we found in our sample might be explained by different prey experience, as we collected the lizards from different areas. In some cases, the direction of laterality can switch from side to side too, as when altering the fear during the test or manipulating sexual motivation (Vallortigara and Andrew, 1994a,b; Bisazza et al., 1997a, 1998; Brown et al., 2007). Vallortigara et al. (1999a), in fact, state that the direction of lateralization is not very important, considering that there are only two possibilities, but rather the direction is different for different tasks. An advantage of displaying lateralized responses may be the possibility to perform many vital tasks simultaneously, i.e. preying and controlling the environment, without constraining any of them (Rogers, 2000; Dadda and Bisazza, 2006).

In conclusion, our study demonstrates how males of Podarcis muralis are lateralized at individual level. The direction of lateralization in a predatory task is the right-hand side, at population level, confirming previous reports in similar contexts. Moreover, this is the first evidence, to our knowledge, of visual lateralization in a lacertid lizard, confirming how lateralization is a widespread phenomenon among the ectotherms; it also supports the hypothesis that vertebrate lateralization is inherited from a common ancestor and preserved during evolution as advantageous character.

\section{Acknowledgements}

We thank Prof. Orazio Rossi for his advice in statistical analyses. This study was supported by Italian Ministero dell'Università e Ricerca and by doctoral grants from the University of Parma to B.B.

\section{References}

Andrew, R.J., 1983. Lateralization of cognitive function in higher vertebrates, with special reference to the domestic chick. In: Ewert, J.-P., Capranica, R.R., Ingle, D. (Eds.), Advances in Vertebrate Neuroethology. Plenum Press, New York, pp. 447-509.

Andrew, R.J., Dharmaretnam, M., 1993. Lateralization and strategies of viewing in the domestic chick. In: Zeigler, H.P., Bischof, H.-J. (Eds.), Vision, Brain and Behaviour in Birds. MIT Press, Cambridge, MA, pp. 319-332.

Bisazza, A., Facchin, L., Pignatti, R., Vallortigara, G., 1998. Lateralization of detour behaviour in poeciliid fish: the effect of species, gender and sexual motivation. Behav. Brain Res. 91, 157-164.

Bisazza, A., Pignatti, R., Vallortigara, G., 1997a. Detour tests reveal task and stimulusspecific behavioural latralization in mosquitofish (Gambusia holbrooki). Behav. Brain Res. 89, 237-242.

Bisazza, A., Pignatti, R., Vallortigara, G., 1997b. Lateralization of detour behaviour: interspecific variation in poeciliid fishes. Anim. Behav. 54, 1273-1281.

Bishop, Y.M.M., Fienberg, S.E., Holland, P.W., 1975. Discrete multivariate analysis. In: Theory and Practice. MIT Press, Cambridge, London, p. 557.

Brown, C., Western, J., Braithwaite, V.A., 2007. The influence of early experience on and inheritance of, cerebral lateralization. Anim. Behav. 74, 231-238.

Butler, A.B., Northcutt, G., 1971. Ascending tectal efferent projections in the lizard Iguana iguana. Brain Res. 35, 597-601.

Cooper Jr., W.E., 2000. An adaptive difference in the relationship between foraging mode and responses to prey chemicals in two congeneric scindid lizards. Ethology 106, 193-206.

Dadda, M., Bisazza, A., 2006. Does brain asymmetry allow efficient performance of simultaneous tasks? Anim Behav. 72, 523-529.

Deckel, A.W., 1995. Laterality of aggressive response in Anolis. J. Exp. Zool. 272, 194-200.

Hews, D.K., Worthington, R.A., 2001. Fighting from the right side of the brain: left visual field preference during aggression in free-ranging male tree lizards (Urosaurus ornatus). Brain Behav. Evol. 58, 356-361.

Hews, D.K., Castellano, M., Hara, E., 2004. Aggression in females is also lateralized: left-eye bias during aggressive courtship rejection in lizards. Anim. Behav. 68, 1201-1207.

McKeehan, A., Sievert, L.M., 1996. The effect of shielding the parietal eye of Podarcis muralis on behavioral thermoregulation. J. Therm. Biol. 21, 397-401.

Robins, A., Rogers, L.J., 2004. Lateralized prey-catching responses in the cane toad Bufo marinus: analysis of complex visual stimuli. Anim. Behav. 68, 567-575.

Robins, A., Rogers, L.J., 2006. Complementary and lateralized forms of processing in Bufo marinus for novel and familiar prey. Neurobiol. Learn. Mem. 86, 214-227.

Robins, A., 2005. Lateralized visual processing in anurans: new vistas though ancient eyes. Eurekah Biosci. 1, 462-473.

Robins, A., Chen, P., Beazley, L.D., Dunlop, S.A., 2005. Lateralized predatory responses in the ornate dragon lizard (Ctenophorus ornatus). Neuroreport 16, 849-852.

Rogers, L.J., 2000. Evolution of hemispheric specialisation: advantages and disadvantages. Brain Lang. 73, 236-253.

Rogers, L.J., Zappia, J.V., Bullock, S.P., 1985. Testosterone and eye-brain asymmetry for copulation in chickens. Experientia 41, 1447-1449.

Sherry, D.F., Schachter, D.L., 1987. The evolution of multiple memory systems. Psycol. Rev. 94, 439-454.

Shine, R., 2003. Locomotor speeds of gravid lizards: placing "costs of reproduction" within an ecological context. Funct. Ecol. 17, 526-533.

SPSS Inc., 2006. SPSS 14.0 for Windows: Base, Professional Statistics and Advanced Statistics. SPSS Inc., Chicago.

Vallortigara, G., Andrew, R.J., 1994a. Olfactory lateralization in the chick. Neuropsycologia 32, 417-423.

Vallortigara, G., Andrew, R.J., 1994b. Differential involvement of right and left hemisphere in individual recognition in the domestic chick. Behav. Process. 33, 41-58.

Vallortigara, G., Bisazza, A., 2002. How ancient is brain lateralization? In: Rogers, L.J. Andrew, R.J. (Eds.), Comparative Vertebrate Lateralization. Cambridge University Press, Cambridge, pp. 9-69.

Vallortigara, G., Rogers, L.J., 2005. Survival with asymmetrical brain: advantages and disadvantages of cerebral lateralization. Behav. Brain Sci. 28, 575-633.

Vallortigara, G., Rogers, L.J., Bisazza, A., 1999a. Possible evolutionary origins of cognitive brain lateralization. Brain Res. Rev. 30, 164-175.

Vallortigara, G., Regolin, L., Pagni, P., 1999b. Detour behaviour, imprinting and visual lateralization in chicks. Cognitive Brain Res. 7, 307-320.

Vallortigara, G., Rogers, L.J., Bisazza, A., Lippolis, G., Robins, A., 1998. Complementary right and left hemifield use for predatory and agonistic behaviour in toads. Neuroreport 9, 3341-3344. 